University of Wollongong

Research Online

Faculty of Informatics - Papers (Archive)

Faculty of Engineering and Information

Sciences

January 2002

\title{
Generalised minimum variance control of linear time-varying systems
}

\section{Zheng Li}

University of Wollongong, zli@uow.edu.au

R. J. Evans

University of Melbourne

Follow this and additional works at: https://ro.uow.edu.au/infopapers

Part of the Physical Sciences and Mathematics Commons

\section{Recommended Citation}

Li, Zheng and Evans, R. J.: Generalised minimum variance control of linear time-varying systems 2002. https://ro.uow.edu.au/infopapers/198

Research Online is the open access institutional repository for the University of Wollongong. For further information contact the UOW Library: research-pubs@uow.edu.au 


\title{
Generalised minimum variance control of linear time-varying systems
}

\author{
Abstract \\ The problem of generalised minimum variance control of linear time-varying discrete-time systems is \\ studied. Standard time-varying controlled autoregressive moving average models are considered, and the \\ sum of plant output tracking error variance plus a penalty term on plant input is chosen as the cost \\ functional. The time-varying controller described is able to minimise the generalised tracking error \\ variance and guarantees closed-loop exponential stability for a large class of linear time-varying systems, \\ including plants which have long time delays and are not stably invertible. \\ Disciplines \\ Physical Sciences and Mathematics \\ Publication Details \\ This paper originaly appeared as: Li, Z, and Evans, RJ, Generalised minimum variance control of linear \\ time-varying systems, IEE Proceedings - Control Theory and Applications, January 2002, 149(1), 111-116. \\ Copyright IEEE 2002.
}




\title{
Generalised minimum variance control of linear time-varying systems
}

\author{
Z.Li and R.J.Evans
}

\begin{abstract}
The problem of generalised minimum variance control of linear time-varying discretetime systems is studied. Standard time-varying controlled autoregressive moving average models are considered, and the sum of plant output tracking error variance plus a penalty term on plant input is chosen as the cost functional. The time-varying controller described is able to minimise the generalised tracking error variance and guarantees closed-loop exponential stability for a large class of linear time-varying systems, including plants which have long time delays and are not stably invertible.
\end{abstract}

\section{Introduction}

The generalised minimum variance controller (GMVC) for single-input single-output linear time-invariant (LTI) plants was developed by Clarke and Gawthrop [1,2], as an extension of the minimum variance controller (MVC) of Astrom [3]. The GMVC for multi-input multi-output LTI systems was proposed by Koivo [4], as an extension of the MVC for multi-input multi-output LTI systems of Borison [5].

These GMVCs are very useful in process control and have seen many applications. In a large number of applications the plant parameters undergo unknown time variations, and a time-varying GMVC is needed for parameter adaptive control in order to minimise a generalised variance cost functional. The purpose of this paper is to extend the GMVCs from the LTI cases to linear timevarying (LTV) systems. A GMVC for LTV plants having coloured noise and a large input/output time delay will be developed through extension.of our previous results on the MVC for LTV systems [6,7], and the GMVC for LTV plants with unit time delay [8].

\section{Mathematical preliminaries}

In this Section we extend our pseudocommutation technique [6] for overcoming noncommutativity problems which arise in the analysis of an LTV GMVC. Consider the LTV moving-averaging autoregressive (MAAR) model:

$$
\begin{aligned}
& \tilde{N}\left(k, q^{-1}\right) v(k)=r(k) \\
& y(k)=\tilde{H}\left(k, q^{-1}\right) v(k)
\end{aligned}
$$

where $q^{-1}$ is the one-step-delay operator, $r(k)$ and $y(k)$ are the plant input and output, $v(k)$ is an unmeasurable internal

(C) IEE, 2002

IEE Proceedings online no. 20020254

DOI: 10.1049/ip-cta:20020254

Paper first received 10th May and in revised form 19th November 2001

$\mathrm{Z}$. $\mathrm{Li}$ is with the School of Electrical, Computer and Telecommunication Engineering, University of Wollongong, Northfields Avenue, Wollongong, NSW 2522, Australia

R.J. Evans is with the Department of Electrical and Electronic Engineering, University of Melbourne, Parkville, Victoria 3052, Australia

IEE Proc.Control Theory Appl., Vol. 149, No. 1, January 2002 state, and $\tilde{N}\left(k, q^{-1}\right)$ and $\tilde{H}\left(k, q^{-1}\right)$ are LTV moving average operators (MAOs) with the forms

$$
\begin{gathered}
\tilde{N}\left(k, q^{-1}\right)=1+\tilde{h}_{1}(k) q^{-1}+\tilde{n}_{2}(k) q^{-2}+\cdots+\tilde{n}_{n}(k) q^{-n} \\
\tilde{H}\left(k, q^{-1}\right)=\tilde{h}_{0}(k)+\tilde{h}_{1}(k) q^{-1}+\tilde{h}_{2}(k) q^{-2}+\cdots+\tilde{h}_{m}(k) q^{-m}
\end{gathered}
$$

It is assumed that their time-varying coefficients are all uniformly bounded away from infinity. The zero initial condition solution to the autoregressive equation can be written as

$$
v(k)=\tilde{N}^{-1}\left(k, q^{-1}\right) r(k)
$$

where $\tilde{N}^{-1}\left(k, q^{-1}\right)$ is called an LTV autoregressive operator (ARO).

Without loss of generality we assume $n \geq m$ and use $\tilde{h}_{i}(\cdot)=0, i=m+1, m+2, \ldots, n$, whenever appropriate. The LTV MAAR model has an $n$-step reachable canonical state space realisation

$$
\begin{aligned}
& \tilde{X}(k+1)=\tilde{\Phi}(k+1, k) \tilde{X}(k)+\tilde{B}(k) r(k) \\
& y(k)=\tilde{C}(k) \tilde{X}(k)+\tilde{D}(k) r(k)
\end{aligned}
$$

where

$$
\begin{aligned}
& \tilde{\Phi}(k+1, k)= \\
& {\left[\begin{array}{cccccc}
-\tilde{n}_{1}(k) & -\tilde{n}_{2}(k) & \cdots & -\tilde{n}_{n-2}(k) & -\tilde{n}_{n-1}(k) & -\tilde{n}_{n}(k) \\
1 & 0 & \cdots & 0 & 0 & 0 \\
0 & 1 & \cdots & 0 & 0 & 0 \\
& & \cdots & & & \\
0 & 0 & \cdots & 0 & 1 & 0
\end{array}\right]} \\
& \tilde{B}(k)=\left[\begin{array}{lllll}
1 & 0 & 0 & \cdots & 0
\end{array}\right]^{\tau}, \quad \tilde{D}(k)=\tilde{h}_{0}(k) \\
& \tilde{C}(k)=\left[\tilde{h}_{1}(k)-\tilde{n}_{1}(k) \tilde{h}_{0}(k), \tilde{h}_{2}(k)-\tilde{n}_{2}(k) \tilde{h}_{0}(k), \ldots,\right. \\
& \left.\tilde{h}_{n}(k)-\tilde{n}_{n}(k) \tilde{h}_{0}(k)\right]
\end{aligned}
$$

The ARO is exponentially stable if and only if the state transition matrix $\tilde{\Phi}(k+1, k)$ is exponentially stable. 
For the LTV MAO pair $\tilde{N}\left(k, q^{-1}\right)$ and $\tilde{H}\left(k, q^{-1}\right)$ we define the following right time-varying Sylvester matrix at time $k$ :

$$
\begin{aligned}
& \tilde{S}_{\tilde{N} \tilde{H}}(k)=\left[\begin{array}{cccc}
1 & 0 & \cdots & 0 \\
\tilde{n}_{1}(k-1) & 1 & \ldots & \ldots \\
\tilde{n}_{2}(k-1) & \tilde{n}_{1}(k-2) & \ldots & 0 \\
\ldots & \ldots & \ldots & 1 \\
\tilde{n}_{n}(k-1) & \tilde{n}_{n-1}(k-2) & \ldots & \tilde{n}_{1}(k-m) \\
0 & \tilde{n}_{n}(k-2) & \ldots & \ldots \\
\ldots & \ldots & \ldots & \tilde{n}_{n-1}(k-m) \\
0 & \ldots & 0 & \tilde{n}_{n}(k-m)
\end{array}\right. \\
& \left.\begin{array}{cccc}
\tilde{h}_{0}(k-1) & 0 & \ldots & 0 \\
\tilde{h}_{1}(k-1) & \tilde{h}_{0}(k-2) & \ldots & \ldots \\
\ldots & \ldots & \ldots & \ldots \\
\tilde{h}_{m}(k-1) & \tilde{h}_{m-1}(k-2) & \ldots & 0 \\
0 & \tilde{h}_{m}(k-2) & \ldots & \tilde{h}_{0}(k-n) \\
\ldots & 0 & \ldots & \ldots \\
\ldots & \ldots & \ldots & \tilde{h}_{m-1}(k-n) \\
0 & \ldots & 0 & \tilde{h}_{m}(k-n)
\end{array}\right]
\end{aligned}
$$

which has the same structure as the time-varying Sylvester matrix described in [6], except for different time indicies in the elements. In the remaining part of this paper we call the Sylvester matrix developed in [6] a left time-varying Sylvester matrix, which is related to reachability. The following lemma shows that the right Sylvester matrix is related to observability.

Lemma 1: The $n$-step reachable canonical form (4) for the LTV MAAR model (1) is $n$-step observable at time $k-n$ if and only if the determinant of the right time-varying Sylvester matrix (7) satisfies

$$
\left|\operatorname{det} \tilde{S}_{\tilde{N} \tilde{H}}(k)\right| \geq \rho
$$

for some constant $\rho>0$.

Clearly, this result is a natural extension of the relation between coprimeness and observability in the LTI case. The proof is omitted here because it is tedious and follows a similar procedure to that given in [6] for reachability. When the $n$-step reachable canonical form is uniformly $n$-step observable it can be uniformly transformed into an $n$-step observable canonical form [9]. By following a similar method to that given in [6] and using a different formulation in the LTV transfer operator framework we have the following.

Lemma 2: If (8) is satisfied for all $k$ and some constant $\rho>0$, then an LTV MAO pair

$$
\begin{aligned}
& N\left(k, q^{-1}\right)=1+n_{1}(k) q^{-1}+n_{2}(k) q^{-2}+\cdots+n_{n}(k) q^{-n} \\
& H\left(k, q^{-1}\right)=h_{0}(k)+h_{1}(k) q^{-1}+h_{2}(k) q^{-2}+\cdots+h_{m}(k) q^{-m}
\end{aligned}
$$

exists such that

$$
N\left(k, q^{-1}\right) \tilde{H}\left(k, q^{-1}\right)=H\left(k, q^{-1}\right) \tilde{N}\left(k, q^{-1}\right)
$$

Coefficients of the LTV MAO's are all uniformly bounded away from infinity and can be uniquely determined using

$$
\begin{gathered}
\tilde{S}_{\tilde{N} \tilde{H}}(k) Z(k)=\tilde{L}(k) \\
h_{0}(k)=\tilde{h}_{0}(k)
\end{gathered}
$$

where

$$
\begin{aligned}
& Z(k)= {\left[\begin{array}{lllll}
h_{1}(k) & h_{2}(k) & \ldots & h_{m}(k) \\
-n_{1}(k) & -n_{2}(k) & \ldots & -n_{n}(k)
\end{array}\right]^{\tau} } \\
& \tilde{L}(k)=\left[\begin{array}{rrrrr}
\tilde{h}_{1}(k)-\tilde{n}_{1}(k) \tilde{h}_{0}(k), \tilde{h}_{2}(k)-\tilde{n}_{2}(k) \tilde{h}_{0}(k), \\
\ldots, \tilde{h}_{n}(k)-\tilde{n}_{n}(k) \tilde{h}_{0}(k) & 0 & 0 & \ldots & 0
\end{array}\right]^{\tau} \quad(13)
\end{aligned}
$$

This lemma is now used to establish the following result on pseudocommutation.

Theorem 1: If the condition in (8) is satisfied for all $k$ and some constant $\rho>0$, then the following properties hold for the LTV MAO pairs $\left\{H\left(k, q^{-1}\right), N\left(k, q^{-1}\right)\right\}$ and $\left\{\tilde{H}\left(k, q^{-1}\right)\right.$, $\left.\tilde{N}\left(k, q^{-1}\right)\right\}$.

P1: Input/output equivalence in the sense that

$$
N^{-1}\left(k, q^{-1}\right) H\left(k, q^{-1}\right)=\tilde{H}\left(k, q^{-1}\right) \tilde{N}^{-1}\left(k, q^{-1}\right)
$$

P2: $N^{-1}\left(k, q^{-1}\right)\left(H^{-1}\left(k, q^{-1}\right)\right)$ is exponentially stable if and only if $N^{-1}\left(k, q^{-1}\right)\left(\tilde{H}^{-1}\left(k, q^{-1}\right)\right)$ is exponentially stable.

P3: The determinant of the left time-varying Sylvester matrix of the LTV MAO pair $N\left(k, q^{-1}\right)$ and $H\left(k, q^{-1}\right)$ satisfies

$$
\left|\operatorname{det} S_{N H}(k)\right| \geq \rho
$$

for all $k$ and some constant $\rho>0$.

P4: $\left|n_{n}(k)\right| \geq c$ and $\left|h_{m}(k)\right| \geq c$ for all $k$ and some constant $c>0$, if and only if $\left|\tilde{n}_{n}(k)\right| \geq \tilde{c}$ and $\left|\tilde{h}_{m}(k)\right| \geq \tilde{c}$ for all $k$ and some constant $\tilde{c}>0$.

Proof: The proof of $\mathrm{P} 1$ and $\mathrm{P} 2$ follows from methods similar to [6]. For P3, letting $\tilde{M}_{R}(k)$ and $\tilde{M}_{O}(k)$ be the $n$-step reachability and observability matrices for the $n$-step-reachable canonical form (4)-(6) and $M_{R}(k)$, and $M_{O}(k)$ be the $n$-step reachability and observability matrices for the corresponding $n$-step-observable form of the same system, we have

$$
\frac{\left|\operatorname{det} M_{R}(k)\right|}{\left|\operatorname{det} \tilde{M}_{R}(k)\right|}=\frac{\left|\operatorname{det} \tilde{M}_{O}(k)\right|}{\left|\operatorname{det} M_{O}(k)\right|}
$$

From [6] we know that

$$
\operatorname{det} S_{N H}(k-n-1)=\operatorname{det} M_{R}(k)
$$

Similarly, it can be shown that

$$
\left|\operatorname{det} \tilde{S}_{\tilde{N} \tilde{H}}(k+n)\right|=\operatorname{det} \tilde{M}_{O}(k)
$$

Noting that $\operatorname{det} M_{O}(k)=\operatorname{det} \tilde{M}_{R}(k)=1$, we have P3.

We use contradiction for the proof of P4. Assume $h_{m}(k)=0$. Expanding both sides of $(10)$ as polynomials in $q^{-1}$ and equating the coefficients of the highest power term we have

$$
n_{n}(k) \tilde{h}_{m}(k-n)=h_{m}(k) \tilde{n}_{n}(k-m)
$$

Because both $\tilde{n}_{n}(k-m)$ and $\tilde{h}_{m}(k-n)$ are uniformly bounded away from zero and all the parameters in the above equation are uniformly bounded away from infinity, it follows from $h_{m}(k)=0$ that $n_{n}(k)=0$. However, the last

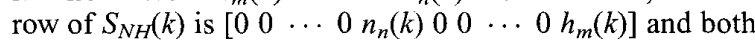
$n_{n}(k)$ and $h_{m}(k)$ are zero, which implies det $S_{N H}(k)=0$. This contradicts P3, and hence P4 is established.

The left-hand side of (14) represents an operation for the zero initial condition solution of an LTV autoregressive moving average (ARMA) model, and we call it an LTV ARMA operator. On the right-hand side of (14), because the

IEE Proc.-Control Theory Appl., Vol. 149, No. 1, January 2002 
order for the moving average and the autoregressive operation is reversed we call it an LTV MAAR operator. In other words, the above pseudocommutation transforms an LTV MAAR operator into an LTV ARMA operator. In contrast, our previous pseudocommutation [6] transforms an LTV ARMA operator into an LTV MAAR operator. In order to distinguish between these two pseudocommutations, we call the pseudocommutation given in theorem 1 right pseudocommutation and our previous pseudocommutation left pseudocommutation. P3 shows that if an LTV MAAR model can be transformed uniquely into an LTV ARMA model by using the right pseudocommutation, then the LTV ARMA model can be uniquely transformed back into the same MAAR model by using the left pseudocommutation, and vice versa. $\mathrm{P} 2$ and $\mathrm{P} 4$ imply that both models have the same exponential stability and the same orders.

\section{Control objective}

We consider the standard LTV controlled ARMA (CARMA) model given by

$$
A\left(k, q^{-1}\right) y(k+d)=B\left(k, q^{-1}\right) u(k)+C\left(k, q^{-1}\right) w(k+d)
$$

where $\{u(k)\}$ and $\{y(k)\}$ are the plant input and output sequence, $d>0$ is an integer representing the minimum delay between the plant input and output, and $\{w(k)\}$ is a zero mean, independent and possibly nonstationary Gaussian sequence with unknown and uniformly bounded variance. The LTV MAO's have the forms:

$$
\begin{aligned}
& A\left(k, q^{-1}\right)=1+a_{1}(k) q^{-1}+a_{2}(k) q^{-2}+\cdots+a_{n}(k) q^{-n} \\
& B\left(k, q^{-1}\right)=b_{0}(k)+b_{1}(k) q^{-1}+b_{2}(k) q^{-2}+\cdots+b_{m}(k) q^{-m} \\
& C\left(k, q^{-1}\right)=1+c_{1}(k) q^{-1}+c_{2}(k) q^{-2}+\cdots+c_{h}(k) q^{-h}(21)
\end{aligned}
$$

We assume the following.

A1: $C^{-1}\left(k, q^{-1}\right)$ is exponentially stable.

A2: Coefficients of $A\left(k, q^{-1}\right), B\left(k, q^{-1}\right)$ and $C\left(k, q^{-1}\right)$ are all uniformly bounded away from infinity, and $b_{0}(k)$ is uniformly bounded away from zero.

A3: The determinants of the left Sylvester matrices of the MAO pair $\left[A\left(k, q^{-1}\right), C\left(k, q^{-1}\right)\right]$ and $\left[B\left(k, q^{-1}\right), C\left(k, q^{-1}\right)\right]$ are uniformly bounded away from zero.

Given a uniformly bounded reference sequence $\{s(k)\}$, the objective is to design a controller which guarantees closed-loop exponential stability and minimises the following generalised output tracking cost functional:

$$
\begin{aligned}
J(k+d)= & E\left\{\left|P\left(k, q^{-1}\right) y(k+d)-R\left(k, q^{-1}\right) s(k)\right|^{2}\right. \\
& \left.+\left|\Lambda\left(k, q^{-1}\right) u(k)\right|^{2} / D(k)\right\}
\end{aligned}
$$

where $D(k)=\{y(k) y(k-1) \ldots, u(k), u(k-1), \ldots\}$ is the set of input and output data up to and including time $k$. The weighting LTV MAO's have the form:

$$
\begin{aligned}
& P\left(k, q^{-1}\right)=1+p_{1}(k) q^{-1}+p_{2}(k) q^{-2}+\cdots+p_{\mu}(k) q^{-\mu} \\
& R\left(k, q^{-1}\right)=r_{0}(k)+r_{1}(k) q^{-1}+r_{2}(k) q^{-2}+\cdots+r_{y}(k) q^{-v} \\
& \Lambda\left(k, q^{-1}\right)=\lambda_{0}(k)+\lambda_{1}(k) q^{-1}+\lambda_{2}(k) q^{-2}+\cdots+\lambda_{\xi}(k) q^{-\xi}
\end{aligned}
$$

They are chosen such that the following assumption is satisfied.

IEE Proc.-Control Theory Appl, Vol. 149, No. 1, January 2002
A4: Coefficients of $P\left(k, q^{-1}\right), R\left(k, q^{-1}\right)$ and $\Lambda\left(k, q^{-1}\right)$ are all uniformly bounded away from infinity, $\lambda_{0}(k)$ is uniformly bounded away from zero, and the determinants of the right time-varying Sylvester matrices for the LTV MAO pair $\left[A\left(k, q^{-1}\right), P\left(k, q^{-1}\right)\right]$ and $\left[B\left(k, q^{-1}\right), \Lambda\left(k, q^{-1}\right)\right]$ are uniformly bounded away from zero.

A4 is an extra assumption imposed on the LTV GMVC compared with the LTI GMVC. In the LTI case, A4 reduces to coprimeness of the time-invariant polynomial pairs $\left[A\left(q^{-1}\right), P\left(q^{-1}\right)\right]$ and $\left[B\left(q^{-1}\right), \Lambda\left(q^{-1}\right)\right]$. In the LTV case, we know from lemma 1 that it is equivalent to the condition that the $n / \xi$-step-reachable canonical realisation of the LTV MAAR operators $P\left(k, q^{-1}\right) A^{-1}\left(k, q^{-1}\right)$ and $B\left(k, q^{-1}\right) \Lambda^{-1}\left(k, q^{-1}\right)$ are uniformly $n / \xi$-step-observable. This assumption is not restrictive because the choice of the weighting LTV MAO's is in our hands.

\section{Generalised minimum variance control}

Without loss of generality we assume that all initial conditions are independent of the process disturbance $\{w(k)\}$. Letting

$$
\psi(k+d)=P\left(k, q^{-1}\right) y(k+d)
$$

be the filtered output in the tracking performance objective, and applying the left pseudocommutation

$$
\begin{aligned}
A\left(k, q^{-1}\right) \tilde{C}\left(k, q^{-1}\right) & =C\left(k, q^{-1}\right) \tilde{A}\left(k, q^{-1}\right) \\
B\left(k, q^{-1}\right) \tilde{D}\left(k, q^{-1}\right) & =C\left(k, q^{-1}\right) \tilde{B}\left(k, q^{-1}\right)
\end{aligned}
$$

we have the following prediction form of the LTV CARMA model

$$
\begin{gathered}
{\left[\begin{array}{cc}
\tilde{C}\left(k-d, q^{-1}\right) & 0 \\
0 & \tilde{D}\left(k, q^{-1}\right)
\end{array}\right]\left[\begin{array}{l}
x(k) \\
z(k)
\end{array}\right]=\left[\begin{array}{l}
y(k) \\
u(k)
\end{array}\right]} \\
\psi(k+d)=\left[G\left(k, q^{-1}\right) F\left(k, q^{-1}\right) \tilde{B}\left(k, q^{-1}\right)\right]\left[\begin{array}{c}
x(k) \\
z(k)
\end{array}\right] \\
+F\left(k, q^{-1}\right) w(k+d)
\end{gathered}
$$

where $\{x(k)\}$ and $\{z(k)\}$ are unmeasurable internal state sequences

$P\left(k, q^{-1}\right) \tilde{C}\left(k, q^{-1}\right)=F\left(k, q^{-1}\right) \tilde{A}\left(k, q^{-1}\right)+G\left(k, q^{-1}\right) q^{-d}$

$F\left(k, q^{-1}\right)=1+f_{1}(k) q^{-1}+\cdots+f_{d-1}(k) q^{-d+1}$.

Noting A1-A3, we know that all coefficients of the LTV MAO's in the prediction model are uniformly bounded away from infinity and the LTV ARO's $\tilde{C}^{-1}\left(k, q^{-1}\right)$ and $\tilde{D}^{-1}\left(k, q^{-1}\right)$ are exponentially stable. It can be shown that $\tilde{B}(k, 0)=b_{0}(k)$ and $\tilde{A}(k, 0)=\tilde{C}(k, 0)=$ $\tilde{D}(k, 0)=1$.

Letting $\hat{\psi}(k+d / k)$ be the $d$-step-ahead prediction of the filtered output $\psi(k)$ based on the plant input and output data up to and including time $k$, and following the method given in [7], we have

$$
\begin{aligned}
& {\left[\begin{array}{cc}
\tilde{C}\left(k-d, q^{-1}\right) & 0 \\
0 & \tilde{D}\left(k, q^{-1}\right)
\end{array}\right]\left[\begin{array}{l}
\hat{x}(k / k) \\
\hat{z}(k / k)
\end{array}\right]=\left[\begin{array}{l}
y(k) \\
u(k)
\end{array}\right](28 a)} \\
& \hat{\psi}(k+d / k)=\left[G\left(k, q^{-1}\right) F\left(k, q^{-1}\right) \tilde{B}\left(k, q^{-1}\right)\right]\left[\begin{array}{l}
\hat{x}(k / k) \\
\hat{z}(k / k)
\end{array}\right]
\end{aligned}
$$


where $\hat{x}(k / k)$ and $\hat{z}(k / k)$ are estimates of the internal states $x(k)$ and $z(k)$. Because of the exponential stability of $\tilde{C}^{-1}\left(k, q^{-1}\right)$ and $\tilde{D}^{-1}\left(k, q^{-1}\right)$, the estimation error for both internal states due to inaccurate initial conditions will exponentially decay to zero, and the prediction of the filtered output will exponentially converge to the minimum variance prediction $\psi(k+d)-F\left(k, q^{-1}\right) w(k+d)$. In the remainder of this paper we shall ignore these exponentially decaying to zero terms caused by inaccurate initial conditions in order to simplify the presentation.

Theorem 2: If assumptions A1-A3 hold for the LTV CARMA model (20), the GMVC which meets the generalised minimum variance control objective (22) is given by

$$
\begin{array}{rc}
{\left[\begin{array}{c}
\tilde{C}\left(k-d, q^{-1}\right) \\
b_{0}(k) G\left(k, q^{-1}\right)
\end{array}\right.} & \left.\begin{array}{c}
b_{0}(k) F\left(k, q^{-1}\right) \tilde{B}\left(k, q^{-1}\right) \\
+\lambda_{0}(k) \Lambda\left(k, q^{-1}\right) \tilde{D}\left(k, q^{-1}\right)
\end{array}\right] \\
\times\left[\begin{array}{c}
\hat{x}(k / k) \\
\hat{z}(k / k)
\end{array}\right] & {\left[\begin{array}{cc}
1 & 0 \\
0 & b_{0}(k) R\left(k, q^{-1}\right)
\end{array}\right]\left[\begin{array}{l}
y(k) \\
s(k)
\end{array}\right]} \\
u(k) & =\left[\begin{array}{ll}
0 & \tilde{D}\left(k, q^{-1}\right)
\end{array}\right]\left[\begin{array}{l}
\hat{x}(k / k) \\
\hat{z}(k / k)
\end{array}\right]
\end{array}
$$

Proof: Substituting $\hat{\psi}(k+d / k)+F\left(k, q^{-1}\right) w(k+d)$ for $\psi(k+d)$ in (22), the generalised minimum variance cost can be rewritten as

$$
\begin{aligned}
J(k+d)= & \left|\hat{\psi}(k+d / k)-R\left(k, q^{-1}\right) s(k)\right|^{2} . \\
& +\left|\Lambda\left(k, q^{-1}\right) u(k)\right|^{2} \\
& +E\left\{\left|F\left(k, q^{-1}\right) w(k+d)\right|^{2} / D(k)\right\}
\end{aligned}
$$

Noting (28b) we have

$$
\begin{aligned}
\partial \hat{\psi}(k+d / k) / \partial u(k) & =[\partial \hat{\psi}(k+d / k) d \hat{z}(k / k)] /[\partial \hat{z}(k / k) d u(k)] \\
& =b_{0}(k)
\end{aligned}
$$

It follows that

$$
\begin{gathered}
\partial J(k+d) / \partial u(\vec{k})=2 b_{0}(k)\left[\hat{\psi}(k+d / k)-R\left(k, q^{-1}\right) s(k)\right] \\
+2 \lambda_{0}(k) \Lambda\left(k, q^{-1}\right) u(k) \\
\partial^{2} J(k+d) / \partial u^{2}(k)=2 b_{0}^{2}(k)+2 \lambda_{0}^{2}(k)
\end{gathered}
$$

Thus, the control sequence which meets the cost in (22) satisfies

$b_{0}(k) \hat{\psi}(k+d / k)+\lambda_{0}(k) \Lambda\left(k, q^{-1}\right) u(k)=b_{0}(k) R\left(k, q^{-1}\right) s(k)$

Substituting $(28 b)$ we have

$$
\begin{aligned}
& \lambda_{0}(k) \Lambda\left(k, q^{-1}\right) u(k)+b_{0}(k) G\left(k, q^{-1}\right) \hat{x}(k / k) \\
& +b_{0}(k) F\left(k, q^{-1}\right) \tilde{B}\left(k, q^{-1}\right) \hat{z}(k / k) \\
& \quad=b_{0}(k) R\left(k, q^{-1}\right) s(k)
\end{aligned}
$$

Noting $(28 a)$ we have the generalised minimum variance control law.

Compared with the LTV MVC our GMVC has an additional term $\left.\lambda_{0}(k) \Lambda\left(k, q^{-1}\right) \tilde{D}\left(k, q^{-1}\right)\right) \hat{z}(k / k)$ on the left-hand side of $(29 a)$, which represents the control weighting $\Lambda\left(k, q^{-1}\right)$. If $\lambda_{0}(k)=0$, the LTV GMVC reduces to the LTV MVC. The LTV GMVC has a different structure from the LTI GMVC. The control variable is computed in two steps. The estimates of the two internal states are generated via the autoregression of the plant output and the reference sequence. The control variable is then determined by a moving average operation. This structure is due to the noncommutativity of the LTV MAO's.

When assumption A4 holds, letting

$$
Q\left(k, q^{-1}\right)=\lambda_{0}(k) \Lambda\left(k, q^{-1}\right)
$$

we introduce the following right pseudocommutation for the analysis of the closed-loop behaviour of the LTV GMVC

$$
\begin{aligned}
& \bar{P}\left(k, q^{-1}\right) A\left(k, q^{-1}\right)=\bar{A}\left(k, q^{-1}\right) P\left(k, q^{-1}\right) \\
& \bar{Q}\left(k, q^{-1}\right) B\left(k, q^{-1}\right)=\bar{B}\left(k, q^{-1}\right) Q\left(k, q^{-1}\right)
\end{aligned}
$$

Substituting $\hat{\psi}(k+d / k)$ using $\psi(k+d)-F\left(k, q^{-1}\right) w(k+d)$ in (34), and noting (24), we have

$$
\begin{aligned}
& b_{0}(k) P\left(k, q^{-1}\right) y(k+d)+Q\left(k, q^{-1}\right) u(k) \\
& \quad=b_{0}(k) F\left(k, q^{-1}\right) w(k+d)+b_{0}(k) R\left(k, q^{-1}\right) s(k)
\end{aligned}
$$

Left multiplying $\bar{B}\left(k, q^{-1}\right)$ and noting the right pseudocommutation (37) we have

$$
\begin{aligned}
\bar{B}(k, & \left.q^{-1}\right) b_{0}(k) P\left(k, q^{-1}\right) y(k+d)+\bar{B}\left(k, q^{-1}\right) Q\left(k, q^{-1}\right) u(k) \\
= & \bar{B}\left(k, q^{-1}\right) b_{0}(k) P\left(k, q^{-1}\right) y(k+d) \\
& +\bar{Q}\left(k, q^{-1}\right) B\left(k, q^{-1}\right) u(k) \\
= & \bar{B}\left(k, q^{-1}\right) b_{0}(k) F\left(k, q^{-1}\right) w(k+d) \\
& +\bar{B}\left(k, q^{-1}\right) b_{0}(k) R\left(k, q^{-1}\right) s(k)
\end{aligned}
$$

Using the LTV CARMA model (20) it follows that

$$
\begin{aligned}
\bar{B}(k, & \left.q^{-1}\right) b_{0}(k) P\left(k, q^{-1}\right) y(k+d) \\
& +\bar{Q}\left(k, q^{-1}\right) A\left(k, q^{-1}\right) y(k+d) \\
& -\bar{Q}\left(k, q^{-1}\right) C\left(k, q^{-1}\right) w(k+d) \\
= & \bar{B}\left(k, q^{-1}\right) b_{0}(k) F\left(k, q^{-1}\right) w(k+d) \\
& +\bar{B}\left(k, q^{-1}\right) b_{0}(k) R\left(k, q^{-1}\right) s(k)
\end{aligned}
$$

Left multiplying $\bar{A}\left(k, q^{-1}\right) b_{0}^{-1}(k)$ on both sides of (38) and then using the right pseudocommutation (37) and CARMA model (20), we have

$$
\begin{aligned}
\bar{P}(k, & \left.q^{-1}\right) B\left(k, q^{-1}\right) u(k)+\bar{P}\left(k, q^{-1}\right) C\left(k, q^{-1}\right) w(k+d) \\
& +\bar{A}\left(k, q^{-1}\right) b_{0}^{-1}(k) Q\left(k, q^{-1}\right) u(k) \\
= & \bar{A}\left(k, q^{-1}\right) F\left(k, q^{-1}\right) w(k+d) \\
& +\bar{A}\left(k, q^{-1}\right) R\left(k, q^{-1}\right) s(k)
\end{aligned}
$$

Combining (40) and (41) we have the following closedloop system:

$$
\begin{aligned}
& {\left[\begin{array}{cc}
\bar{A}\left(k, q^{-1}\right) b_{0}^{-1}(k) Q\left(k, q^{-1}\right) & 0 \\
+\bar{P}\left(k, q^{-1}\right) B\left(k, q^{-1}\right) & \bar{Q}\left(k, q^{-1}\right) A\left(k, q^{-1}\right) \\
0 & +\bar{B}\left(k, q^{-1}\right) b_{0}(k) P\left(k, q^{-1}\right)
\end{array}\right]} \\
& \quad \times\left[\begin{array}{c}
u(k) \\
y(k+d)
\end{array}\right] \\
& =\left[\begin{array}{cc}
\bar{A}\left(k, q^{-1}\right) R\left(k, q^{-1}\right) & \bar{A}\left(k, q^{-1}\right) F\left(k, q^{-1}\right) \\
\bar{B}\left(k, q^{-1}\right) b_{0}(k) R\left(k, q^{-1}\right) & \bar{Q}\left(k, q^{-1}\right) C\left(k, q^{-1}\right) \\
& +\bar{B}\left(k, q^{-1}\right) b_{0}(k) F\left(k, q^{-1}\right)
\end{array}\right] \\
& \quad \times\left[\begin{array}{c}
s(k) \\
w(k+d)
\end{array}\right]
\end{aligned}
$$

The LTV MAO matrix on the left-hand side of the closedloop system has a diagonal structure, and stability of the closed-loop system is determined by the LTV ARO's, $\left[\bar{A}\left(k, q^{-1}\right) b_{0}^{-1}(k) Q\left(k, q^{-1}\right)+\bar{P}\left(k, q^{-1}\right) B\left(k, q^{-1}\right)\right]^{-1}$ and

IEE Proc.-Control Theory Appl., Vol. 149, No. 1, January 2002 
$\left[\bar{Q}\left(k, q^{-1}\right) A\left(k, q^{-1}\right)+\bar{B}\left(k, q^{-1}\right) b_{0}(k) P\left(k, q^{-1}\right)\right]^{-1}$. If the LTV AROs are exponentially stable and the input signals $s(k)$ and $w(k+d)$ are zero, then $y(k+d)$ and $u(k)$ will decay to zero exponentially. Noting (26a) and (28a) we know that the internal states and their estimates, i.e. $x(k), z(k), \hat{x}(k / k)$ and $\hat{z}(k / k)$, will all decay to zero exponentially due to the exponential stability of the LTV ARO's $\tilde{C}^{-1}\left(k, q^{-1}\right)$ and $\tilde{D}^{-1}\left(k, q^{-1}\right)$. Thus, the overall closed-loop system including all the internal variables is exponentially stable.

Theorem 3: When assumptions $\mathrm{A} 2$ and $\mathrm{A} 4$ hold the LTV ARO $\left[\bar{A}\left(k, q^{-1}\right) b_{0}^{-1}(k) Q\left(k, q^{-1}\right)+\bar{P}\left(k, q^{-1}\right) B\left(k, q^{-1}\right)\right]^{-1}$ is exponentially stable if and only if the LTV ARO $[\bar{Q}(k$, $\left.\left.q^{-1}\right) A\left(k, q^{-1}\right)+\bar{B}\left(k, q^{-1}\right) b_{0}(k) P\left(k, q^{-1}\right)\right]^{-1}$ is exponentially stable.

Proof: Without loss of generality we assume $n \geq \mu$ and consider an exponentially stable closed-loop system

$$
\left[\bar{A}\left(k, q^{-1}\right) b_{0}^{-1}(k) Q\left(k, q^{-1}\right)+\bar{P}\left(k, q^{-1}\right) B\left(k, q^{-1}\right)\right] u(k)=0
$$

Introducing internal states

$$
\begin{aligned}
& \bar{x}(k)=B\left(k, q^{-1}\right) u(k) \\
& b_{0}(k) \bar{z}(k)=-Q\left(k, q^{-1}\right) u(k)
\end{aligned}
$$

Equation 43 can be rewritten as

$$
\begin{gathered}
\bar{z}(k)=\bar{A}^{-1}\left(k, q^{-1}\right) \bar{P}\left(k, q^{-1}\right) \bar{x}(k) \\
\bar{x}(k)=-B\left(k, q^{-1}\right) Q^{-1}\left(k, q^{-1}\right) b_{0}(k) \bar{z}(k)
\end{gathered}
$$

The closed-loop system can be considered as the LTV ARMA model ( $45 a$ ) under the control of the LTV MAAR feedback controller (45b) as shown in Fig. 1. Noting $u(k)$ decays exponentially to zero and the coefficients of both $B\left(k, q^{-1}\right)$ and $Q\left(k, q^{-1}\right)$ are all uniformly bounded away from infinity, we know from (44) that $\bar{x}(k)$ and $\bar{z}(k)$ decay to zero at a rate faster than or equal to the rate of $u(k)$. Using right pseudocommutation (37), the above closed-loop system is equivalent to

$$
\begin{array}{r}
\bar{z}(k)=P\left(k, q^{-1}\right) A^{-1}\left(k, q^{-1}\right) \bar{x}(k) \ldots \\
\bar{x}(k)=-\bar{Q}^{-1}\left(k, q^{-1}\right) \bar{B}\left(k, q^{-1}\right) b_{0}(k) \bar{z}(k)
\end{array}
$$

which can be further rewritten as

$$
\begin{gathered}
\bar{z}(k)=P\left(k, q^{-1}\right) \bar{r}(k) \\
A\left(k, q^{-1}\right) \bar{r}(k)=\bar{x}(k) \\
\bar{Q}\left(k, q^{-1}\right) \bar{x}(k)=-\bar{B}\left(k, q^{-1}\right) b_{0}(k) \bar{z}(k)
\end{gathered}
$$

where $\bar{r}(k)$ is an internal state. (47) can be considered as a closed-loop system where the LTV MAAR model ( $47 a$ and $47 b$ ) is regulated by the ARMA model feedback controller $(47 c)$ as shown in Fig. 2. Substituting (47a) and (47b) into $(47 c)$ we have

$$
\left[\bar{Q}\left(k, q^{-1}\right) A\left(k, q^{-1}\right)+\bar{B}\left(k, q^{-1}\right) b_{0}(k) P\left(k, q^{-1}\right)\right] \bar{r}(k)=0
$$

Noting A4 and following the method given in [6], it can be shown that the $n$-step reachable canonical form of (47a) and $(47 b)$ is uniformly $n$-step observable. It follows that $\bar{r}(k)$ decays exponentially to zero at a rate faster than or equal to that of $z(k)$. Thus, the closed-loop system in (48) is exponentially stable with a rate faster than or equal to the rate of the closed-loop system in (43). Similarly, it can be shown that when the closed-loop system in (48) is expo-

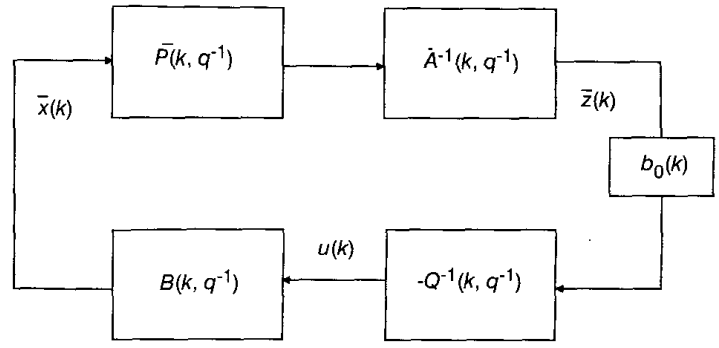

Fig. 1 Closed-loop system of LTV GMVC

nentially stable, the closed-loop system in (43) is exponentially stable with a rate faster than or equal to the rate of (48).

Theorem 3 shows that for the analysis of closed-loop stability, only one of the two LTVAROs in (42) needs to be checked.

\section{Simulation}

We consider a first-order LTV plant given by

$$
\begin{aligned}
y(k+2)+ & a(k) y(k+1)=u(k) \\
& +b(k) u(k-1)+w(k+2)+c(k) w(k+1)
\end{aligned}
$$

where $w(k)$ is an independent stationary gaussian noise with zero mean and unit variance. The reference output is a zero mean square wave. The plant parameters are

$$
\begin{aligned}
& a(k)=-2+0.8 \cos (0.1 k \pi+0.2 \pi), \quad b(k)=3 \\
& c(k)=\left\{\begin{array}{cc}
0.9 \frac{k+2}{k+3} & 20(2 i-1)+3<k \leq 40 i+3 \\
-0.9 \frac{k+2}{k+3} & 40 i+3<k \leq 20(2 i+1)+3
\end{array}\right.
\end{aligned}
$$

where $i$ is an integer. Obviously, the LTV ARO's $A^{-1}(k$, $\left.q^{-1}\right)$ and $B^{-1}\left(k, q^{-1}\right)$ are exponentially unstable while $C^{-1}\left(k, q^{-1}\right)$ is exponentially stable. The weighting LTV MAOs are

$$
\begin{aligned}
P\left(k, q^{-1}\right) & =1+p(k) q^{-1} \\
& =1+[0.0667-0.03 \cos (0.1 k \pi+0.2 \pi)] q^{-1} \\
\Lambda\left(k, q^{-1}\right) & =1+\lambda(k) q^{-1} \\
& =1+0.1 q^{-1}, \quad R\left(k, q^{-1}\right)=0.7
\end{aligned}
$$

While the LTV MAO $R\left(k, q^{-1}\right)$ does not affect closed-loop stability, and can be chosen freely, the LTV MAO's $P\left(k, q^{-1}\right)$ and $\Lambda\left(k, q^{-1}\right)$ must be chosen in such a way that they result in a stable closed-loop system. Applying

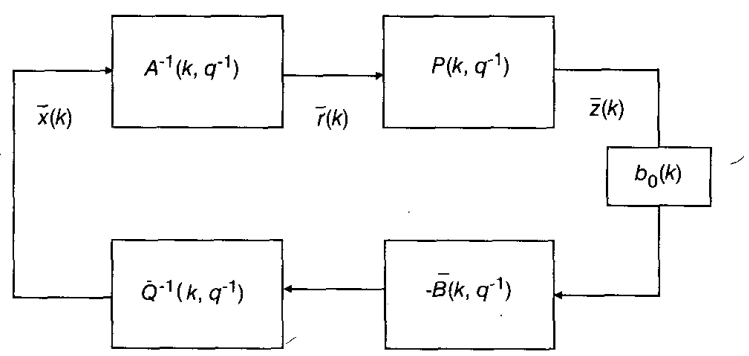

Fig. 2 An equivalent closed-loop system for the LTV GMVC 
the right pseudocommutation and using theorem 3, we know that closed-loop stability is determined by

$$
\begin{aligned}
& \bar{Q}\left(k, q^{-1}\right) A\left(k, q^{-1}\right)+\bar{B}\left(k, q^{-1}\right) b_{0}(k) P\left(k, q^{-1}\right) \\
& \quad=2\left[1+\frac{1.17+0.773 \cos (0.1 k \pi+0.2 \pi)}{2} q^{-1}\right]
\end{aligned}
$$

Noting that

$$
\left|\frac{1.17+0.773 \cos (0.1 k \pi+0.2 \pi)}{2}\right|<0.98
$$

we know that the closed-loop system is exponentially stable.

Using the left transformation

$$
\tilde{f}(\alpha(k), \beta(k))=\frac{\alpha(k+1)[\beta(k)-\alpha(k)]}{\beta(k+1)-\alpha(k+1)}
$$

the plant (49) and (50) can be rewritten in prediction form, where

$$
\begin{aligned}
& \tilde{a}(k)=\tilde{f}(a(k), c(k)), \tilde{b}(k)=\bar{f}(b(k), c(k)) \\
& \tilde{c}(k)=\bar{f}(c(k), a(k)), \tilde{d}(k)=\bar{f}(c(k), b(k))
\end{aligned}
$$

with the subscript 1 suppressed for simplicity.

Applying theorem 2 for a two-step-ahead LTV GMVC, we have

$$
\begin{gathered}
\hat{x}(k / k)+\tilde{c}(k-2) \hat{x}(k-1 / k-1)=y(k) \\
2 \hat{z}(k / k)+\left[f_{1}(k)+\lambda(k)+\tilde{b}(k)+\tilde{d}(k)\right] \hat{z}(k-1 / k-1) \\
+\left[f_{1}(k) \tilde{b}(k-1)+\lambda(k) \tilde{d}(k-1)\right] \hat{z}(k-2 / k-2) \\
=-g_{0}(k) \hat{x}(k / k)+0.7 s(k) \\
\quad u(k)=\hat{z}(k / k)+\tilde{d}(k) \hat{z}(k-1 / k-1)
\end{gathered}
$$

where

$$
\begin{aligned}
& f_{1}(k)=p(k)+c(k)-a(k) \\
& g_{0}(k)=[p(k)-a(k)][c(k-1)-a(k-1)]
\end{aligned}
$$

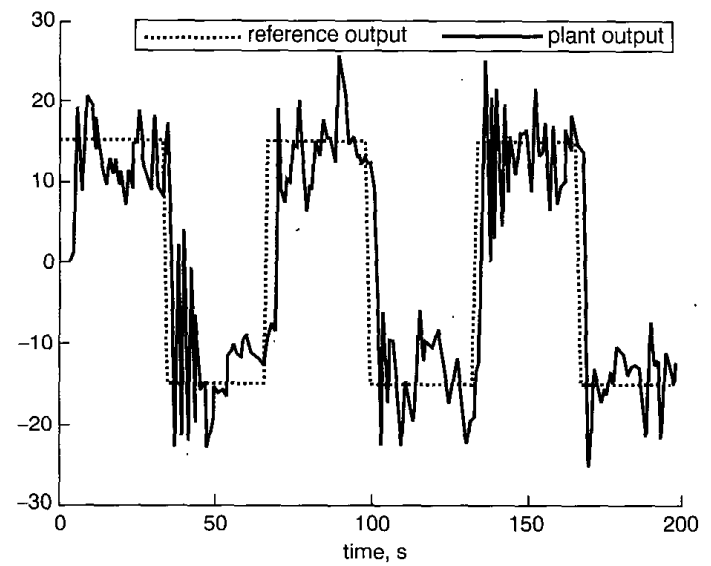

Fig. 3 Performance of LTV GMVC

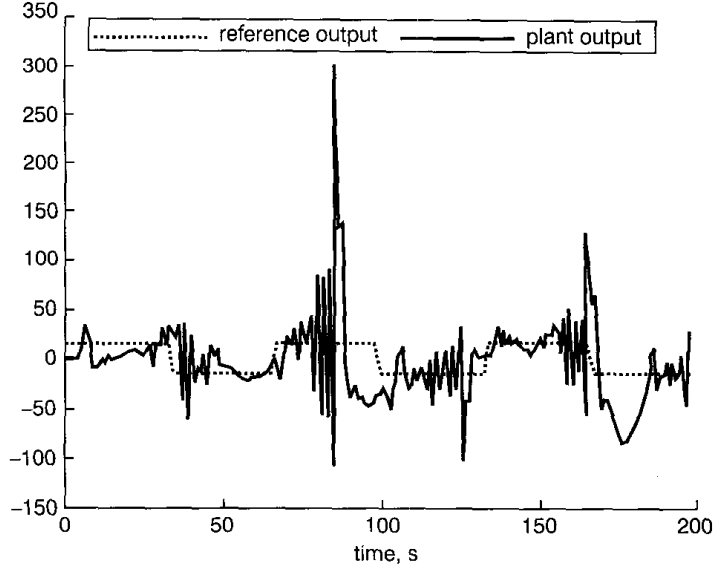

Fig. 4 Performance of LTI model-based GMVC

Fig. 3 shows that our LTV GMVC works well for this example. The LTI GMVC is also applicable to the same system in an indirect adaptive control scheme as follows. On each sampling instant the current plant parameters are used for the design of the LTI GMVC as if the plant were LTI. This procedure is repeated for each sampling instant, resulting in an LTV controller based on the LTI GMVC design. Fig. 4 shows that this LTI GMVC-based LTV controller causes large oscillations in the plant output with an amplitude up to 20 times the reference.

\section{Conclusions}

An LTV GMVC has been developed for a large class of LTV systems described using the standard LTV CARMA model, allowing both rapid time variations in the plant parameters and long input/output delay. When compared with the LTV MVC, the LTV GMVC has improved robustness and flexibility. In particular, the exponentially stable invertibility is no longer necessary for closed-loop stability.

\section{References}

1 CLARKE, D.W., and GAWTHROP, P.J.: 'Self-tuning controller', IEE Proc. D, 1975, 122, pp. 929-934

2 CLARKE, D.W., and GAWTHROP, P.J.: 'Self-tuning control', IEE Proc. $D, 1979$, 133, pp. 633-640

3 ASTROM, K.J.: 'Introduction to stochastic control theory' (Academic Press, New York, 1970)

4 KOIVO, H.N.: 'A multivariable self-tuning controller', Automatica, $1980,16, p p .351-366$

5 BORISON, U.: 'Self-tuning regulators for a class of multivariable systems', Automatica, 1979, 15, pp. 209-215

6 LI, Z., and EVANS, R.J.: 'Minimum variance control of linear timevarying systems', Automatica, 1997, 33, pp. 1531-1537

7 LI, Z., EVANS, R.J., and WITTENMARK, B.: 'Minimum variance prediction for linear time-varying systems', Automatica, 1997, 33, pp. $607-618$

8 LI, Z.: 'An adaptive controller for time-varying systems'. Proceedings of international conference (Control '88), University of Oxford, UK, 1988 international con $523-526$

9 pp. 523-526 Sohn, Braunschweig, Wiesbaden, 1981) 